\title{
ANÁLISIS FUNCIONAL DE CRECIMIENTO Y DESARROLLO DE CUATRO VARIEDADES DE PAPA (Solanum tuberosum subsp. andigena)
}

Fecha de recepción: Junio 15 de 2013 • Fecha de aceptación: Julio 28 de 2013

\section{GROWTH AND DEVELOPMENT FUNCTIONAL ANALYSIS IN FOUR POTATO VARIETIES (Solanum tuberosum subsp. andigena)}

Ángela Patricia Gaitán Moreno • María Paulina Alejandra González Mogollón² • Carlos Eduardo Nústez López ${ }^{3}$ Tatiana María Saldaña Villota ${ }^{4}$ José Miguel Cotes Torres ${ }^{5}$

\section{RESUMEN}

Bajo las condiciones ambientales de la Sabana de Bogotá, se realizó un análisis de crecimiento funcional en las variedades de papa Monserrate, Única, Zipa y Morita pertenecientes a la especie Solanum tuberosum subsp. andigena. Se establecieron parcelas únicas de $90 \mathrm{~m}^{2}$ a una densidad de 33.333 plantas ha-1. Semanalmente se evaluó el peso seco por órgano y el área foliar a partir de la semana siete, en dos plantas por variedad. Se calculó la tasa relativa de crecimiento, tasa de crecimiento del cultivo, relación área foliar, tasa de asimilación neta, área foliar específica e índice de área foliar. La variedad Única tuvo el mejor rendimiento por planta, no almacena fotoasimilados de forma temporal en los tallos y el llenado de los tubérculos tiene una duración de 12 semanas. La variedad Monserrate tuvo el segundo mejor rendimiento por planta, almacena fotoasimilados temporalmente en los tallos vegetativos durante una semana y el tiempo de llenado de los tubérculos dura 9 semanas. Las variedades Morita y Zipa muestran un comportamiento intermedio en su análisis de crecimiento respecto a las otras variedades estudiadas, sin embargo, en términos de producción, Morita es la variedad que tuvo el menor rendimiento por planta, incluso por unidad de área. Por su parte, Zipa se caracteriza por requerir un área foliar sana y eficiente hasta la semana 16, con base en la cual se obtienen los fotoasimilados necesarios para el llenado de tubérculos. La variedad Morita con los rendimientos por planta más bajos es la que finaliza en menor tiempo el llenado de tubérculos.

Palabras clave: Tasa de Asimilación Neta, Tasa Relativa de Crecimiento, Distribución de Materia Seca.

1. Ingeniera Agrónoma. Facultad de Agronomía. Universidad Nacional de Colombia - Sede Bogotá. Carrera 45 N²6-85.

2. Ingeniera Agrónoma. Facultad de Agronomía. Universidad Nacional de Colombia - Sede Bogotá. Carrera $45 \mathrm{~N}^{\circ} 26-85$.

3. Ingeniero Agrónomo. MSc. DSc. Profesor Asociado. e-mail: cenuztezl@unal.edu.co. Facultad de Agronomía. Universidad Nacional de Colombia - Sede Bogotá. Carrera $45 \mathrm{~N}^{\circ} 26-85$

4. Ingeniera Agrónoma. Maestría en Ciencias Agrarias. e-mail: tmsaldanav@unal.edu,co. Facultad de Ciencias Agrarias. Universidad Nacional de Colombia - Sede Medellín. Calle 59a № 63-20. Nucleo El Volador

5. Ingeniero Agrónomo. MSc. DSc. Profesor Asociado. e-mail: jmcotes@unal.edu.co. Facultad de Ciencias Agrarias. Universidad Nacional de Colombia - Sede Medellín. Calle 59a № 63-20. Nucleo El Volador. 


\section{ABSTRACT}

Under the environmental conditions of the "Sabana de Bogotá, a functional growth analysis on potato varieties (Solanum tuberosum L.) Monserrate, Unica, Zipa and Morita were performed. Experimental units with an area of $90 \mathrm{~m}^{2}$ were established at a density of 33.333 plants ha-1. Weekly, the dry weight by organ and leaf area were evaluated from the seventh week, in two plants per variety. The relative growth rate, crop growth rate, leaf area, net assimilation rate, specific leaf area and leaf area index were calculated. Única variety had the best yield per plant, it does not store the photoassimilates temporarily in the stems and the dry matter allocation to the tubers lasts 12 weeks. Monserrate variety had the second best yield per plant, the photoassimilates stored temporarily in vegetative stems for a week and the dry matter allocation to the tubers lasts 9 weeks. Morita and Zipa varieties showed an intermediate behavior in their growth analysis compared to the other varieties in this study, however, in terms of production, Morita had the lowest yield per plant, even by unit area. Zipa is characterized by requiring a healthy and efficient leaf area leaf area until to week 16 . From this leaf area, the photoassimilates necessary for filling the tubers are obtained. Morita, with lower yields per plant, ends the dry matter allocation to the tubers earliest.

Keywords: Net Assimilation Rate, Relative Growth Rate, Dry Matter Allocation.

\section{INTRODUCCIÓN}

El crecimiento de los vegetales es un proceso que resulta de la interacción entre fotosíntesis, respiración, transporte de asimilados, relaciones hídricas y nutrición mineral; es el incremento irreversible en materia seca, volumen, longitud o área como resultado de la división, expansión y diferenciación celular. El desarrollo, es el conjunto de procesos que determinan el cambio de formas y aptitudes en un individuo (Lambers et al. 1998; Larcher, 2003). El crecimiento y desarrollo están determinados por factores genéticos y ambientales, dentro de los últimos se encuentran variables como la temperatura y la radiación solar, el primero, tiene efecto directo sobre la velocidad de las actividades enzimáticas y el segundo, sobre la actividad fotosintética (Tekalign y Hammes, 2005).

El análisis de crecimiento es una aproximación cuantitativa para comprender el crecimiento de una planta o de una población de plantas bajo condiciones ambientales naturales o controladas. Este crecimiento puede ser cuantificado mediante el empleo de un conjunto de índices basados en modelos definidos mediante expresiones o funciones matemáticas (Hunt, 2003; Kooman y Rabbinge, 1996). Puede ser analizado en términos de un incremento del peso seco total de la planta y su distribución entre sus órganos tanto en la parte aérea como en la subterránea. De este modo, el patrón de asignación de biomasa desempeña un papel fundamental en la toma de nutrientes y por lo tanto a su tasa de crecimiento (Lambers et al. 1998).

El análisis de crecimiento se ha desarrollado de dos formas. La primera denominada análisis clásico, el cual contempla medidas hechas entre intervalos de tiempo largos utilizando un gran número de plantas. La segunda forma se denomina análisis 


\section{El análisis de crecimiento ha sido ampliamente usado para estudiar los factores que influyen en el rendimiento y desarrollo de las plantas como la acumulación de fotoasimilados netos en el tiempo (Gardner et al. 1985).}

funcional, que comprende medidas a intervalos de tiempo más frecuentes usando un pequeño número de plantas. El objetivo principal del análisis funcional del crecimiento vegetal es obtener funciones matemáticas que describan adecuadamente el crecimiento de las plantas (Hunt, 2003). A diferencia del análisis clásico, el análisis funcional utiliza la totalidad de la información colectada en campo para definir cada punto de la curva de crecimiento. Entre las ventajas del análisis funcional se destaca el hecho de que las funciones matemáticas resumen mucha información en pocos parámetros. Al momento de seleccionar el modelo adecuado además del ajuste estadístico se debe dar prioridad al criterio biológico (Hunt, 1982).

Las funciones polinomiales son empleadas frecuentemente para la descripción del crecimiento vegetal. La de primer grado describe una línea recta y es poco utilizada en este tipo de análisis. La de segundo grado o cuadrática, cuya función es $W=a+b_{1} T+b_{2} T^{2}$, donde $W$ es el logaritmo del peso y $T$ es el tiempo; la primera derivada de esta función es corresponde a la Tasa Relativa de Crecimiento (TRC). El parámetro $a$ representaría el tamaño del peso, cuando $T=0, b_{1}$, indica el valor de TRC en el tiempo $T=0$ y $b_{2}$ refleja el grado de curvatura o tasa de cambio en las progresiones de la TRC. La función polinomial de tercer grado o cúbica presenta una función $W=a+b_{1} T+b_{2} T^{2}+b_{3} T^{3}$ y es más utilizada. El parámetro $a$ tiene el mismo significado mencionado anteriormente, pero es difícil asignar un significado biológico a los coeficientes $b_{(.)}$. En este caso la curva será determinada por los signos de los coeficientes y el grado de curvatura por sus magnitudes (Marcelis et al. 1998; Tekalign y Hammes, 2005a, 2005b).

El análisis de crecimiento ha sido ampliamente usado para estudiar los factores que influyen en el rendimiento y desarrollo de las plantas como la acumulación de fotoasimilados netos en el tiempo (Gardner et al. 1985). Este usa medidas directas, como peso seco, área foliar total y tiempo. Las medidas derivadas son calculadas a partir de las medidas directas y corresponden a la tasa relativa de crecimiento (TRC), tasa de crecimiento del cultivo (TCC), relación de área foliar (RAF), tasa de asimilación neta (TAN), área foliar específica (AFE), índice de área foliar (IAF) y duración de área foliar (DAF) (Hunt, 1982).

En Colombia, en el cultivo de papa, se han realizado trabajos que brindan información sobre el crecimiento y desarrollo de la especie. Nústez et al. (2009), evaluaron la acumulación y distribución de materia seca en los órganos de la planta de las variedades Betina, Pastusa Suprema, Esmeralda y DIACOL Capiro, encontrando diferencias en cuanto a la acumulación de materia seca en hojas y tallos en la que sobresale Pastusa Suprema por presentar la mayor acumulación pero el menor índice 
Las variedades de papa (Solanum tuberosum

de cosecha. Segura et al. (2006), evaluaron el desarrollo fenológico de estas mismas variedades y encontraron similitud en la ocurrencia y duración de las etapas fenológicas y duración del ciclo de Esmeralda, Betina y DIACOL Capiro, mientras que Pastusa Suprema tiene un desarrollo fenológico más tardío.

En México, Aguilar et al. (2006), realizaron un análisis de crecimiento y de las relaciones fuente - demanda de las variedades de papa Alpha y Milagros, ajustaron los datos de peso seco de cada órgano a modelos polinomiales y concluyeron que el rendimiento de tubérculos está determinado por el área foliar. Por su parte, Borrego et al. (2000), hicieron un análisis de crecimiento de siete variedades de papa, lo que les permitió detectar los genotipos de papa más eficientes en su acumulación de biomasa. Afirman que los índices de mayor utilidad para establecer las diferencias entre los materiales fueron el IAF y la TAN.

Con en el fin de profundizar en el conocimiento de las variedades de papa en las condiciones agroecológicas de Colombia, el objetivo de este trabajo fue estimar mediante un análisis funcional, el crecimiento y desarrollo de cuatro variedades de papa en condiciones de la Sabana de Bogotá .

\section{MATERIALES Y MÉTODOS}

El trabajo de campo se realizó en el Centro Agropecuario Marengo, de la Universidad Nacional de Colombia, ubicado en el municipio de Mosquera (Cundinamarca) a $23 \mathrm{~km}$ de Bogotá, a una altura de 2650 msnm, sus coordenadas geográficas son $4^{\circ} 42^{\prime} L N$, $74^{\circ} 12^{\prime} \mathrm{LO}$, su temperatura media anual es de $13^{\circ} \mathrm{C}$, se registran valores de precipitación de 528,9 mm anuales y una humedad relativa de $80-85 \%$. De acuerdo a las características de precipitación, temperatura y evapotranspiración la zona se clasifica como Bosque Seco Montano Bajo (bs - MB) (Holdridge, 1967). subsp. andigena), que hicieron parte del estudio fueron ICA Zipa, ICA Única, ICA Morita y DIACOL Monserrate. El experimento se desarrolló en un arreglo espacial de parcelas únicas para cada una de las variedades, la unidad experimental fue un área de 90 $\mathrm{m}^{2}$, estuvo constituida por nueve surcos de $10 \mathrm{~m}$ de largo por $1 \mathrm{~m}$ entre ellos. En cada surco se sembraron 11 tubérculos a 0,30 m. Se realizaron muestreos semanales a partir de la séptima semana después de siembra, cuando el $50 \%$ de las plantas había emergido. Las muestras fueron llevadas a estufas de secado a temperatura constante de $70^{\circ} \mathrm{C}$ durante $72 \mathrm{~h}$.

\section{Análisis Estadístico}

Las variables evaluadas fueron peso seco de hojas $\left(P_{H}\right)$, peso seco de tallos $\left(P_{T}\right)$, peso seco de tubérculos $\left(P_{T B}\right)$ y área foliar $(A F)$, esta última mediante la lectura directa en el planímetro.

Para estas variables, se obtuvieron curvas exponenciales estimadas por regresión lineal mediante transformación logarítmica de la forma

$$
y=\exp \left(\beta_{0}+\beta_{1} t+\beta_{2} t^{2}+\beta_{3} t^{3}+\varepsilon\right)
$$

donde, exp es la constante de Neper (2,71828), $b_{(.)}$son los coeficientes de regresión y $\varepsilon$ es el error experimental o residual.

El peso seco de la planta (P) se obtuvo como la suma de los modelos de los pesos secos de cada órgano

$$
P=\sum_{i} e^{P_{i}} \quad i=\{\text { hojas, tallos, tubérculos }\}
$$

donde $P_{i}$ son las ecuaciones obtenidas para el peso seco de hojas, tallos y tubérculos.

Como medidas de ajuste se evaluaron el coeficiente de variación del modelo y el coeficiente de determinación del mismo para cada una de las variables. 
En el caso del P, se realizó una prueba de $\chi^{2}$ entre el valor estimado por el modelo [2] y los datos observados. Para el análisis se utilizó SAS v. 8.0.

\section{Parámetros de Crecimiento}

Para el cálculo de las tasas relativas de crecimiento (de la planta y de sus órganos) se utilizó la siguiente fórmula:

$$
\begin{aligned}
& T R C_{i}=\frac{1}{P_{i}} \times \frac{d P_{i}}{d t} \\
& T R C_{i}=\hat{\beta}_{1 i}+2 \hat{\beta}_{2 i} t+3 \hat{\beta}_{3 i} t^{2}
\end{aligned}
$$

donde $\frac{d P_{(i)}}{d t}$ es la primera derivada del peso en función del tiempo y $\hat{\beta}_{(.)}$son los coeficientes de regresión estimados para hojas, tallos y tubérculos.

La TRC de la planta es:

$$
T R C=\sum T R C_{i}
$$

La TAC por órgano se calculó de la siguiente forma:

$$
\begin{gathered}
T A C_{i}=\frac{d P_{i}}{d t} \\
T A C_{i}=\exp \left(\hat{\beta}_{0}+\hat{\beta}_{1 i} t+\hat{\beta}_{2 i} t^{2}+\hat{\beta}_{3 i} t^{3}\right)
\end{gathered}
$$

La TAC de la planta se obtuvo mediante la siguiente expresión:

$$
T A C=\sum T A C_{i}
$$

Para obtener la TAN se utilizó:

$$
T A N=\frac{1}{A F} \times \sum_{i} \frac{d P_{i}}{d t}
$$

La RAF se obtuvo como la relación entre el área foliar y el peso seco de la planta; el área foliar específica (AFE) se calculó como cociente entre el área foliar y el peso seco de las hojas y la RPF se obtuvo como el resultado de las hojas entre el peso seco de la planta. Note que $R A F=A F E \times R P F$ y $T R C=T A N \times R A F$.

\section{RESULTADOS Y DISCUSIÓN}

Los modelos encontrados para las variables muestran un buen ajuste $\left(R^{2}>0,80\right)$ (Tabla 1). No hay diferencias estadísticas significativas $(p>0,05)$ entre el peso seco total y el obtenido mediante la suma de los modelos estimados del peso seco de cada órgano (Tabla 2), indicando que el modelo del peso seco total es adecuado.

Tabla 1. Parámetros estadísticos de ajuste de los modelos estimados. ( $R^{2}$ : Coeficiente de determinación. CV: Coeficiente de Variación. P: Peso seco total. AF: Área Foliar. $\mathrm{P}_{\mathrm{H}}$ : Peso Seco de Hojas. $\mathrm{P}_{\mathrm{T}}$ : Peso seco de tallos. $\mathrm{P}_{\mathrm{TB}}$ : Peso seco de tubérculos.

\begin{tabular}{ccccccccc}
\hline & \multicolumn{2}{c}{ Monserrate } & \multicolumn{2}{c}{ Única } & \multicolumn{2}{c}{ Zipa } & \multicolumn{2}{c}{ Morita } \\
\hline $\begin{array}{c}\text { Variable } \\
\text { Dependiente }\end{array}$ & $\mathbf{R}^{\mathbf{2}}$ & $\mathbf{C V}$ & $\mathbf{R}^{\mathbf{2}}$ & $\mathbf{C V}$ & $\mathbf{R}^{\mathbf{2}}$ & $\mathbf{C V}$ & $\mathbf{R}^{\mathbf{2}}$ & $\mathbf{C V}$ \\
\hline $\mathbf{P}$ & 0,9626 & 8,5927 & 0,9811 & 6,1146 & 0,9512 & 9,5244 & 0,9753 & 6,1448 \\
\hline $\mathbf{A F}$ & 0,9160 & 4,6336 & 0,9493 & 2,9576 & 0,8215 & 6,0964 & 0,8098 & 4,2753 \\
\hline $\mathbf{P}_{\mathbf{H}}$ & 0,9474 & 10,9169 & 0,9604 & 9,0175 & 0,8049 & 19,9265 & 0,9218 & 9,4769 \\
\hline $\mathbf{P}_{\mathrm{T}}$ & 0,9657 & 12,9745 & 0,9660 & 13,5168 & 0,9687 & 12,4345 & 0,9730 & 11,5695 \\
\hline $\mathbf{P}_{\mathrm{TB}}$ & 0,9763 & 16,2899 & 0,9530 & 25,9861 & 0,9331 & 30,4766 & 0,9690 & 18,7281 \\
\hline
\end{tabular}


Tabla 2. Prueba de ajuste entre el modelo general de crecimiento de la planta y la suma de los modelos parciales encontrados.

\begin{tabular}{ccc}
\hline Variedad & $\chi^{2}$ & Prob $>\chi^{2}$ \\
\hline Monserrate & 4,043 & 0,997 \\
\hline Única & 9,638 & 0,842 \\
\hline Zipa & 10,987 & 0,753 \\
\hline Morita & 1,314 & 0,999 \\
\hline
\end{tabular}

\section{TASA DE ASIMILACIÓN NETA Y TASA RELATIVA DE CRECIMIENTO}

Se determinó que Monserrate es una variedad de desarrollo tardío en comparación con las demás variedades estudiadas, su TRC llega a cero en la semana 21, contrastando con Única, que se considera una variedad de desarrollo precoz, puesto que su TRC alcanza el cero cuatro semanas antes que Monserrate (Figura 1). Las variedades Zipa y Morita tienen un comportamiento intermedio, en las cuales su TRC llega a cero en la semana 18 y 19 respectivamente. La TRC permite cuantificar la velocidad de crecimiento de los vegetales (Hunt, 2002), en este sentido, Monserrate es la que se destaca como la variedad más eficiente en términos de producción de materia seca a la semana 7 , en ese momento del ciclo incrementa su peso seco por planta en $0,73 \mathrm{~g} \mathrm{~g}^{-1} \mathrm{sem}^{-1}$. Única y Morita, en esta misma semana incrementan su materia seca por planta tan solo 0,63 y 0,62 $\mathrm{g} \mathrm{g}^{-1} \mathrm{sem}^{-1}$, respectivamente. En estas tres variedades, la TRC se reduce a la mitad cuando los valores de la TAN son máximos. Por su parte, la TRC en Morita se comporta diferente respecto a las demás variedades. En esta variedad, la TRC por planta tiene un comportamiento parabólico que inicia en $0,27 \mathrm{~g} \mathrm{~g}^{-1}$ $\mathrm{sem}^{-1}$, asciende hasta $0,46 \mathrm{~g} \mathrm{~g}^{-1} \mathrm{sem}^{-1}$ en la semana 12 y en ese momento empieza a descender. En todas las variedades la TRC es máxima entre las semanas 15 y 16 .

Las variedades Monserrate, Única y Zipa alcanzan los máximos valores de TAN por planta en la semana $15\left(0,014,0,017\right.$ y $0,012 \mathrm{~g} \mathrm{~cm}^{-2} \mathrm{sem}^{-1}$, respectivamente) mientras que Morita lo hace en la semana $16\left(0,019 \mathrm{~g} \mathrm{~cm}^{-2} \mathrm{sem}^{-1}\right)$. En la variedad Monserrate se destaca una etapa donde el valor de TAN presenta valores con pequeños incrementos hasta la semana 16, a partir de la semana 17 los valores comienzan a descender drásticamente. Las variedades Única, Zipa y Morita, también muestran inicialmente estabilidad en los valores de TAN hasta la semana 17 y en esa semana los valores decrecen pero no de manera tan rápida. En Monserrate, Zipa y Morita, la TAN se vuelve cero entre la semanas 18 y 19 indicando que la producción potencial de estas variedades se define entre la semanas 17 y 18 , sin ser las semanas precedentes unas más determinantes que otras por la suave tendencia ascendente presentada. La variedad Única presenta una alta actividad fotosintética entre las semanas 13 y 18 , periodo de tiempo en el cual se presenta un incremento de la TAN que mantiene valores positivos hasta la semana 19.

En los resultados que obtuvieron Condori et al. (2008), en términos de TAN las variedades de papa Luki (S. tuberosum subsp. andigena), Waycha y Alpha (S. tuberosum subsp. tuberosum) son más eficaces, aunque no encontraron diferencias con otras especies que producen tubérculos, obteniendo valores por planta entre 1,05 y $1,40 \mathrm{~g} \mathrm{~cm}^{-1} \mathrm{sem}^{-1}$. Aguilar et al. (2006), obtuvieron valores de TAN en la variedad Milagros del orden de 0,009 $\mathrm{g} \mathrm{cm}^{-2} \mathrm{sem}^{-1}$ $y$ en Alpha de $0,008 \mathrm{~g} \mathrm{~cm}^{-2} \mathrm{sem}^{-1}$ en fases iniciales del cultivo (dos semanas después de la emergencia), coincidiendo con Grime y Hunt (1975) lo que permite concluir que las diferencias varietales y la ubicación geográfica de los experimentos son determinantes en el comportamiento fisiológico de la especie. Por su parte, Lynch y Rowberry (1977), 

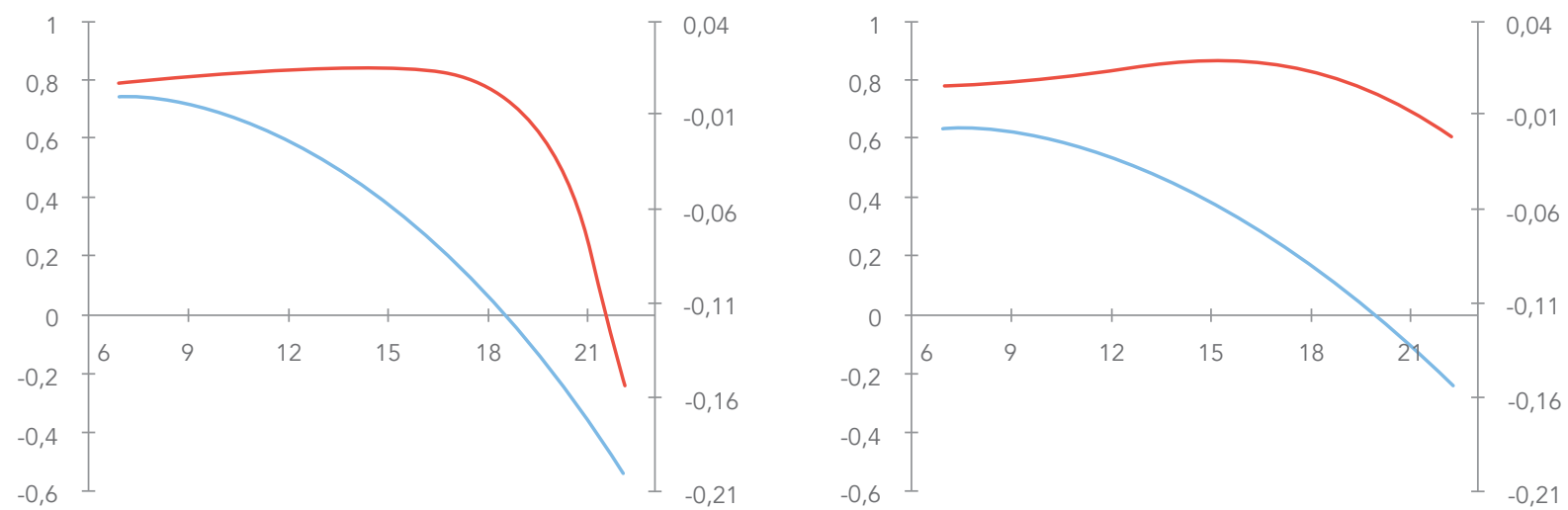

(A)
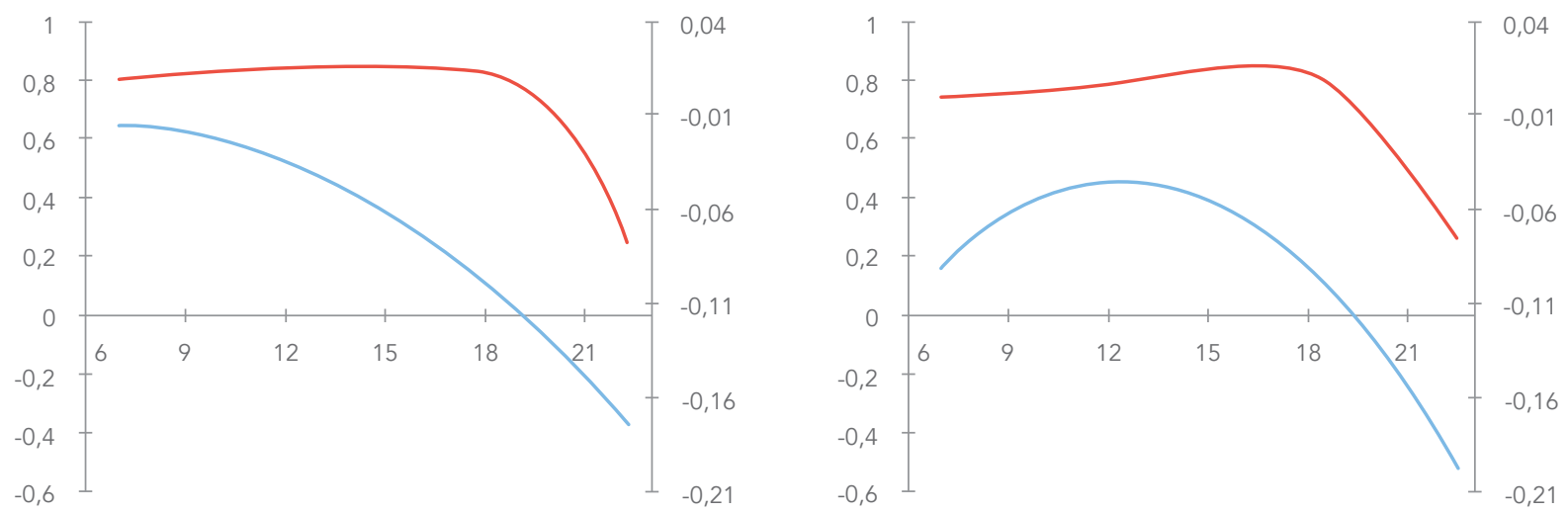

(C)

Figura 1. Tasa de asimilación neta (TAN) y Tasa relativa de crecimiento (TRC). En el eje de las ordenadas de la izquierda está la TRC en $\mathrm{g}^{-1}$ sem $^{-1} \mathrm{y}$ en el de la derecha la TAN en $\mathrm{g} \mathrm{cm}^{-2} \mathrm{sem}^{-1}$. La línea roja indica la TAN, mientras que la línea azul la TRC. A. Variedad Monserrate. B. Variedad Única. C. Variedad Zipa. D. Variedad Morita.

realizaron un análisis de crecimiento de la variedad Russet Burbank en Guelph (Canadá), obteniendo resultados similares en el comportamiento de la TRC y la TAN a los del este trabajo, donde la TRC decrece con el tiempo y alcanza un valor igual a cero cuando la TAN alcanza los máximos valores, es decir, considerando la TAN como estimativa de la fotosíntesis neta, las plantas alcanzan su máximo potencial fotosintético y este no se ve reflejado en su crecimiento, lo que indica una mayor respiración y una menor fotosíntesis bruta en el inicio de la senescencia de la planta.

\section{RELACIÓN ÁREA FOLIAR, ÁREA FOLIAR ESPECÍFICA Y RELACIÓN PESO FRESCO}

La RAF en las variedades Monserrate, Única y Zipa tienen un comportamiento similar que decrece constantemente durante el ciclo del cultivo y sus valores descienden a partir de la semana 15 y 16, respectivamente. La variedad Morita, tiene un comportamiento particular en el que incrementa su RAF hasta la semana 9 y en ese momento empieza a disminuir (Figura 2). En la variedad Monserrate, la RAF está influenciada principalmente por uno de sus 


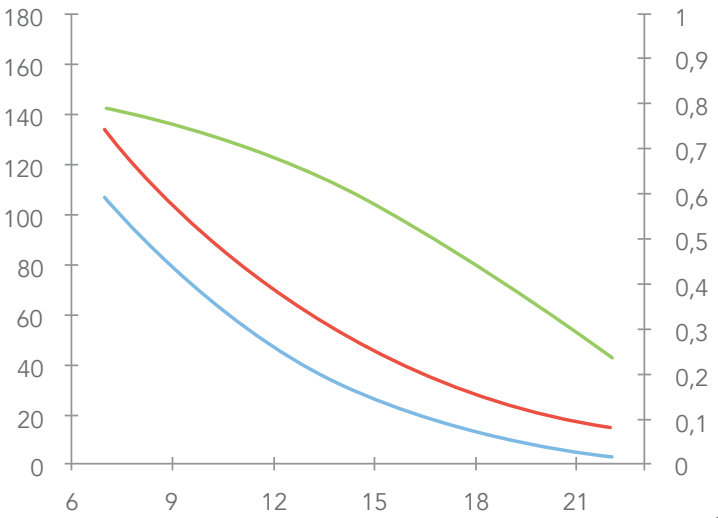

(A)

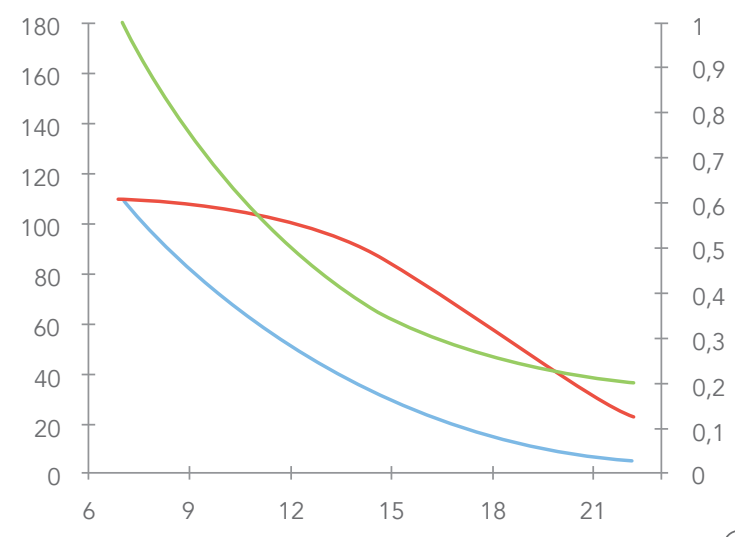

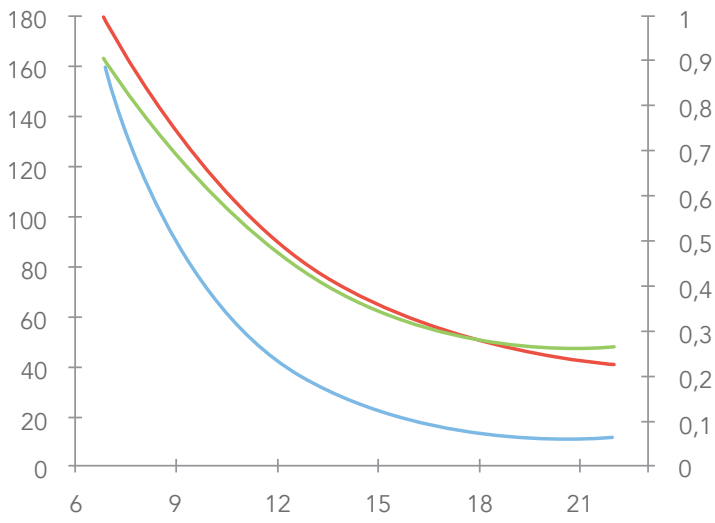

(B)

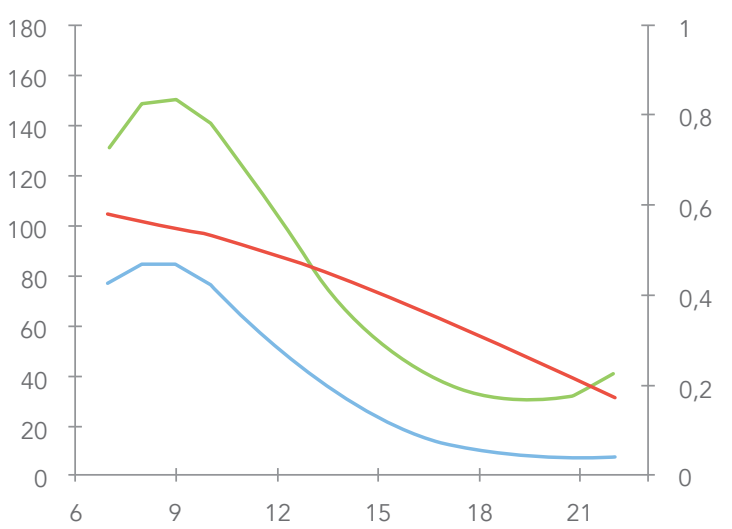

(D)

Figura 2. Razón Área Foliar (RAF) y sus componentes Área Foliar Específica (AFE), Relación Peso Fresco (RPF). En el eje de las ordenadas de la izquierda están RAF y AFE en $\mathrm{cm}^{2} \mathrm{~g}^{-1}$ y en el de la derecha está la RPF en $\mathrm{g} \mathrm{g}^{-1}$. La línea azul indica la RAF, la línea roja indica la AFE y la línea verde indica la RPF. A. Variedad Monserrate. B. Variedad Única. C. Variedad Zipa. D. Variedad Morita.

componentes, el AFE, al presentar ambas variables la misma tendencia. Por lo anterior se puede concluir que esta variedad para mantener su RAF realiza cambios en la densidad de sus hojas y no hace cambios en la distribución de materia seca entre los órganos fotoasimiladores y los demás órganos de la planta. En la variedad Única, la RAF es igualmente afectada por sus dos componentes AFE y RPF por lo cual, la planta recurre a cambios en la densidad de sus hojas y a cambios en la distribución de sus fotoasimilados con el fin de evitar que la TRC decrezca a mayor velocidad. Para las variedades Zipa y Morita, el componente de mayor importancia en la RAF, es la RPF, contrario a la variedad Monserrate. Este comportamiento indica que para mantener la RAF, la plantas recurren a cambios fuertes en la distribución de materia seca.

\section{TASA ABSOLUTA DE CRECIMIENTO}

Las variedades Monserrate y Zipa alcanzan las máximas TAC en la semana 16, mientras que Única y Morita lo hacen en la semana 17. Aunque casi no hay diferencia en el momento en el cual las variedades 
alcanzan ese máximo valor, sí hay diferencia en cuanto a los valores como tal, destacándose Monserrate y Única como las que logran más ganancia de peso seco por planta a través del tiempo (82,82 y 81,82 $\mathrm{g}$ $\mathrm{sem}^{-1}$, respectivamente), seguidas por Zipa (49,36 g $\mathrm{sem}^{-1}$ ) y Morita $\left(34,34 \mathrm{~g} \mathrm{sem}^{-1}\right)$ (Figura 3). Este parámetro permite determinar claramente las variedades que sobresalen por su ganancia de peso en el tiempo. A pesar de que en las semanas 17 y 18 las variedades alcanzan valores máximos de TAC en los tubérculos, sobresale Única, cuya $\mathrm{TAC}_{\mathrm{TB}}$ por planta en ese momento es de 39,14 $\mathrm{g} \mathrm{sem}^{-1}$, seguida de Monserrate y Zipa (16,34 y 14,86 $\mathrm{g} \mathrm{sem}^{-1}$, respectivamente ) y la variedad Morita queda en último lugar con un valor considerablemente bajo de 8,30 $\mathrm{g} \mathrm{sem}^{-1}$, lo que permite afirmar que Morita no tiene una buena producción de tubérculos, debido a que su desarrollo vegetativo es muy pobre, tal vez porque esta variedad está adaptada a otra zona agroecológica.

La variedad Monserrate deja de acumular materia seca inicialmente en hojas y en tallos en la semana 18 y finalmente en tubérculos en la semana 21 . Entre las semanas 14 y 15 la planta tiene la misma TAN, y hace una mayor distribución de fotoasimilados hacia tallos que hacia hojas. En este momento los fotoasimilados no son distribuidos hacia los tubérculos debido a que los tallos presentan mayor tasa de crecimiento $\left(36,65 \mathrm{~g} \mathrm{sem}^{-1}\right)$, coincidiendo con los resultados de Aguilar et al. (2006), donde para las variedades Alpha y Milagros se presenta el mismo comportamiento. Finalmente los fotoasimilados se depositan en los tubérculos obteniendo su máxima tasa de acumulación (16,34 $\mathrm{g} \mathrm{sem}^{-1}$ ) en la semana 18, y finalizando su llenado en la semana 21, confirmando que la producción potencial en esta variedad se define antes de la semana 18. Entre la semana 16 y 19 hay un almacenamiento temporal de los fotoasimilados en los tallos y en la semana 21 se alcanza el llenado completo de los tubérculos (Figura 3A).

La variedad Única, presenta picos de acumulación de materia seca alrededor de la semana 17 (Figura 3B). Son cercanos y poco diferenciados para hojas, tallos y tubérculos, por lo que se asume que en esta variedad no se realiza un almacenamiento temporal de los fotoasimilados y estos van directamente de la fuente a su vertederos, tubérculos o tallos según sea el caso, ya que los dos tienen la misma tasa de crecimiento. Como consecuencia de la ausencia de almacenamiento temporal de fotoasimilados, se recomienda que hasta la semana 21 se realicen controles sanitarios destinados a proteger el área foliar.

En la variedad Zipa, el mayor incremento en la acumulación de materia seca se da en la semana 15 para hojas, semana 16 para tallos y semana 18 para tubérculos (Figura 3C). Se presenta una diferencia de dos semanas entre hojas y tallos, permitiendo un almacenamiento temporal de fotoasimilados en tallos desde donde son dirigidos a los tubérculos

Las variedades Única y Monserrate se presentaron como las mejores alternativas por su elevada producción, sin embargo estas son antagónicas en varios aspectos de su crecimiento. 


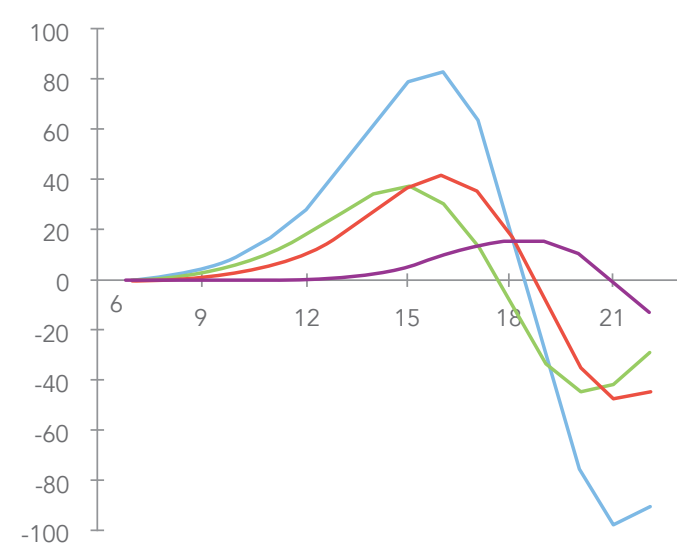

(A)
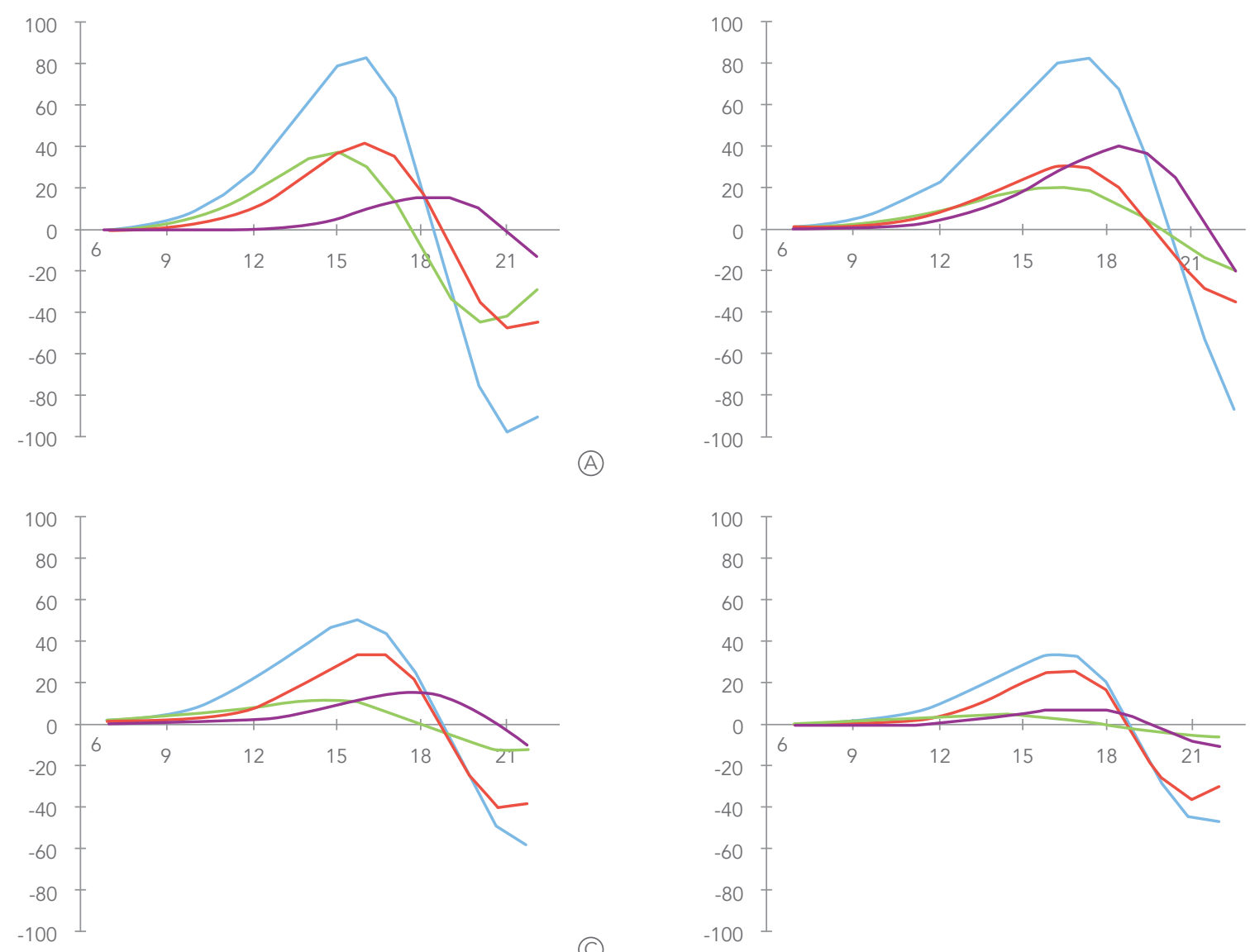

(C)

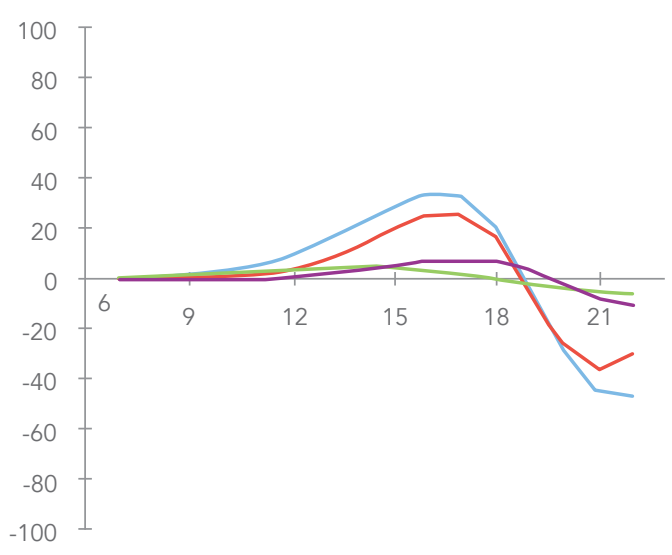

(B)

Figura 3. Tasa absoluta de crecimiento (TAC). En el eje de las ordenadas está la TAC en $\mathrm{g} \mathrm{sem}^{-1}$. La línea azul representa el total de la planta. La línea verde indica las hojas, la línea roja indica los tallos y la línea morada representa los tubérculos. A. Variedad Monserrate. B. Variedad Única. C. Variedad Zipa. D. Variedad Morita.

en las siguientes dos semanas (18 y 19) coincidiendo con la mayor tasa de acumulación de materia seca de los tubérculos por planta $\left(14,86 \mathrm{~g} \mathrm{sem}^{-1}\right)$ y la interrupción del almacenamiento de materia seca en los tallos. Este comportamiento permite concluir que la protección del área foliar es necesaria hasta la semana 16.

En la curva de acumulación de materia seca de la variedad Morita, sobresale el protagonismo de los tallos como principales vertederos de fotoasimilados que alcanzan un valor máximo de TAC en la semana
17 de 27,35 $\mathrm{g} \mathrm{sem}^{-1}$ desde donde después de una semana son traslocados a los tubérculos (Figura 3D). La mayores valores en tasa de acumulación de materia seca para tallos coinciden con la mayores TAN $(0,019$ y $\left.0,018 \mathrm{~g} \mathrm{~cm}^{-2} \mathrm{sem}^{-1}\right)$, entonces, este momento es de especial cuidado para el área foliar.

\section{ACUMULACIÓN DE MATERIA SECA}

La variedad que más materia seca acumula por planta es Única $(475,45$ g), seguida por Monserrate 

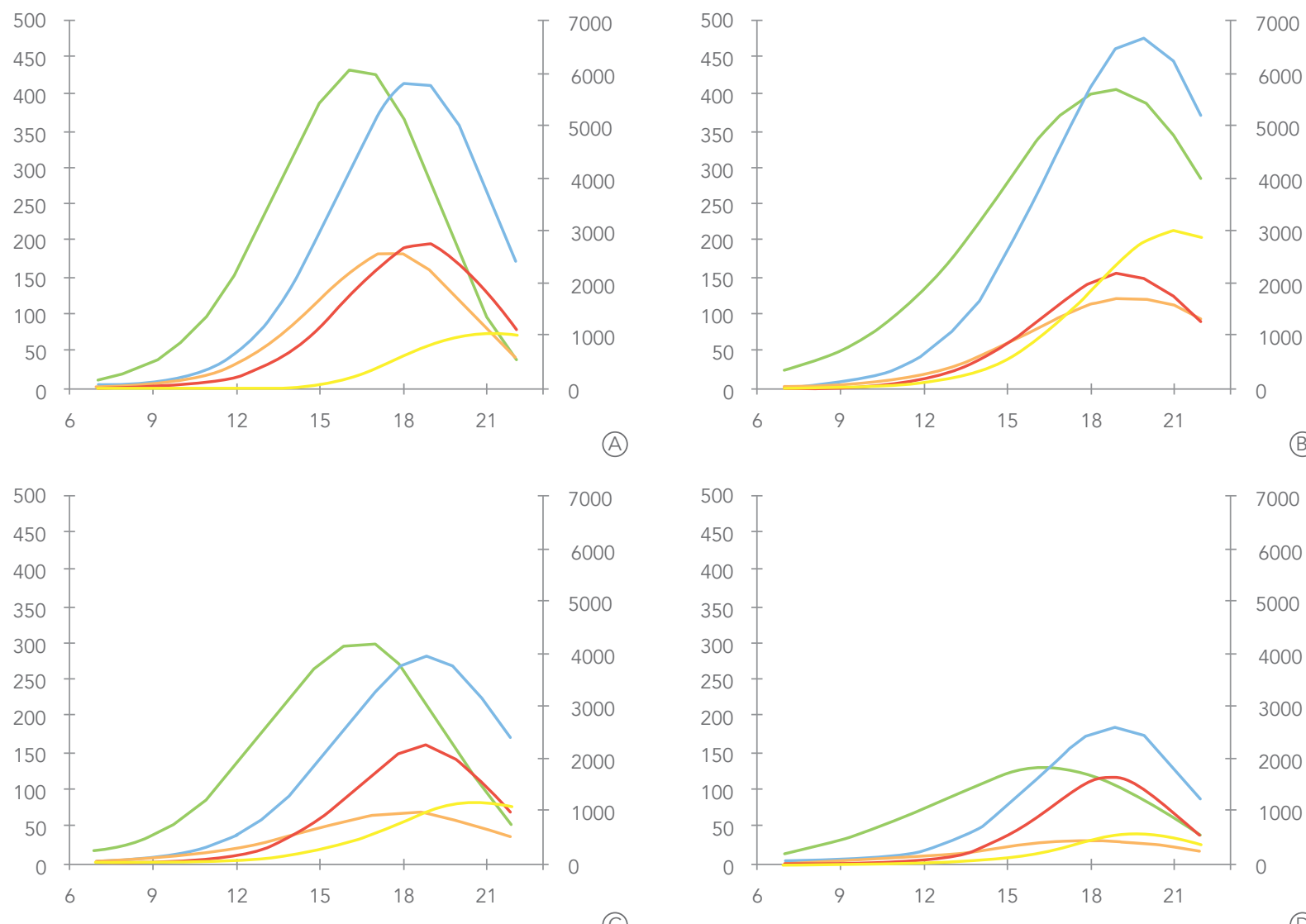

(C)

Figura 4. Curvas de crecimiento para los distintos órganos de la planta en términos de materia seca (MS) y área foliar (AF). En el eje de las ordenadas de la izquierda está MS en g y en el de la derecha está AF en $\mathrm{cm}^{2}$. La línea azul representa el peso seco total (PS), la línea naranja representa el peso seco de hojas (PSH), la línea roja representa el peso seco de tallos (PST), la línea amarilla representa el peso seco de los tubérculos (PSTB) y la línea verde representa el área foliar (AF). A. Variedad Monserrate B. Variedad Única. C. Variedad Zipa. D. Variedad Morita.

(414,63 g), Zipa $(277,27$ g) y Morita (186,34 g) (Figura 4). Sin embargo, Monserrate es la variedad que más rápido alcanza su máximo peso seco en la semana 18, mientras que Única logra su mayor peso seco dos semanas después que Monserrate y una semana después de Zipa y Morita (Figura 4).

Cuando la TAC es máxima, se acumula el $78,1 \%, 70,8 \% 70,6 \%$ y $64,9 \%$ del peso total para las variedades Morita, Única, Monserrate y Zipa, respectivamente. Se observó un rango de acumulación de materia seca total por planta entre 186,34 y 475,45 g en las variedades Morita y Única, respectivamente, entre la semanas 19 y 20 después de emergencia. Estos valores son inferiores a los valores más altos encontrados por Nústez et al. (2009) en las variedades de S. tuberosum spp andigena, Betina, Esmeralda, DIACOL Capiro y Pastusa Suprema (entre 520 y 733 g planta-1) entre las semanas 14 y 18, en un experimento realizado en Zipaquirá (Cundinamarca). Esto confirma, la influencia del ambiente y el genotipo sobre las variables fisiológicas de las plantas. 
Con excepción de la variedad Morita, las variedades en estudio finalizaron maduración de tubérculos en la semana 21, mientras Morita lo hizo una semana antes. La diferencia en el inicio de tuberización es de una semana entre cada variedad, empieza Única en la semana 9, seguida de Zipa, Morita y finalizando con Monserrate en la semana 12.
La variedad que más peso seco alcanza en las hojas es Monserrate (182,18 $\mathrm{g} \mathrm{planta}^{-1}$ ) y lo hace en la semana 18. Se presenta una importante diferencia con la variedad Morita, que logra su máxima acumulación de materia seca en las hojas en esa misma semana pero el valor es apenas de 31,33 $\mathrm{g}$ planta-1 ${ }^{-1}$ demostrando nuevamente que es una variedad cuya producción de biomasa es muy pobre y su potencial de rendimiento para la zona de estudio es bajo.

No hay diferencias importantes en la acumulación máxima de materia seca en los tubérculos entre las variedades Monserrate y Zipa (76,15 y 81,46 g planta $^{-1}$, respectivamente), ambas variedades alcanzan su máximo en la semana 21. En este aspecto, se destaca la variedad Única, que también logra el mayor valor de materia seca en los tubérculos en la semana 21, pero su valor es considerablemente más alto (214,05 g planta $\left.^{-1}\right)$.

La senescencia de la planta se presenta primero para en variedad Monserrate, seguida de Morita, Zipa en la misma semana, y finalmente Única en las semanas 18, 19 y 20, respectivamente (Figura 3). Se hace evidente que la variedad más productiva es la que más tarda en entrar a su periodo senescente, sin embargo, Monserrate que es la segunda en rendimiento por planta es también la segunda en iniciar el proceso de senescencia, lo que constituye una ventaja frente a las otras variedades.

Con excepción de la variedad Morita, las variedades en estudio finalizaron maduración de tubérculos en la semana 21, mientras Morita lo hizo una semana antes. La diferencia en el inicio de tuberización es de una semana entre cada variedad, empieza Única en la semana 9, seguida de Zipa, Morita y finalizando con Monserrate en la semana 12. Las variedades Morita y Monserrate tienen el mismo período de llenado correspondiente a 9 semanas mientras que el periodo de llenado para la variedad Zipa es de 10 semanas y el de la variedad 
Única es de 11 semanas. Así se explica la mayor acumulación de materia seca en los tubérculos de esta última, por la mayor duración en el llenado del tubérculo y por la no utilización de los tallos como almacenamiento temporal, lo que le evita perder materia seca acumulada en procesos de construcción y reconstrucción de carbohidratos.

\section{CONCLUSIONES}

Las variedades Única y Monserrate se presentaron como las mejores alternativas por su elevada producción, sin embargo estas son antagónicas en varios aspectos de su crecimiento. La variedad Única, tuvo el mejor rendimiento por planta, no posee almacenamiento temporal de fotoasimilados en los tallos vegetativos y su llenado de tubérculo toma más tiempo (11 semanas) mientras que Monserrate presenta acumulación de fotoasimilados en tallos durante las semanas 16 a 19 y el llenado de tubérculos lo realiza en 9 semanas.

Las variedades Morita y Zipa muestran comportamiento intermedio en su análisis de crecimiento, sin embargo, en términos de producción Morita es la que tuvo la menor producción, presentando una posible desadaptación a la zona agroecológica evaluada.

En el caso particular de la TAN, se observó que este comportamiento fue similar entre las variedades Morita y Única y entre Monserrate y Zipa lo que significaría producciones potenciales similares, sin embargo, las diferencias que se presentaron se explican por factores como la eficiencia en la distribución de fotoasimilados, potencial de demanda del vertedero y adaptación de las variedades a la zona del estudio.

\section{BIBLIOGRAFÍA}

1. Aguilar, M. G., Carrillo, J., Rivera, A., and González, V. 2006. Growth analysis and sink-source relationships in two potato (Solanum tuberosum L.) varieties. Revista Fitotecnia Mexicana 29(2): 145-156.

2. Borrego, F., Murillo, M., Fernández, J., López, A., Parga, V., and Carvajal, A. 2000. Nota Técnica. Anáisis de crecimiento en siete variedades de papa (Solanum tuberosum L.). Agronomía Mesoaméricana 11(1): 145-149.

3. Condori, B., Mamani, P., Botello, R., Patiño, F., Devaux, A., and Ledent, J. F. 2008. Agrophysiological characterisation and parametrisation of Andean tubers: Potato (Solanum sp.), oca (Oxalis tuberosa), isaño (Tropaeolum tuberosum) and papalisa (Ullucus tuberosus). European Journal of Agronomy 28(4): 526-540.

4. Grime, J., and Hunt, R. 1975. Relative growth-rate: Its range and adaptive significance in a local flora. Journal of Ecology 63(2): 393-422.

5. Hunt, R. 2002. A Modern Tool for Classical Plant Growth Analysis. Annals of Botany 90(4): 485-488.

6. Hunt, R. 2003. Growth analysis, individual plants. Sheffield. Elsevier. UK.

7. Kooman, P. L., and Rabbinge, R. 1996. An analysis of the relation between dry matter allocation to the tuber and earliness of a potato crop. Annals of Botany 77: 235-242.

8. Lambers, H., Chapin, F. S., and Pons, T. 1998. Plant Physiological Ecology. (Second Edition., p. 604). Springer. U.S.A. 
9. Larcher, W. 2003. Physiological plant ecology. Ecophysiology and stress physiology of the functional groups. Vol. 93, p. 513. Springer. Berlin.

10. Lynch, D. R., and Rowberry, R. G. 1977. Population density studies with Russet Burbank II. The effect of fertilization and plant density on growth, development and yield. American Potato Journal 54: 57-71.

11. Marcelis, L. F., Heuvelink, E., and Goudriaan, J. 1998. Modelling biomass production and yield of horticultural crops: a review. Scientia Horticulturae 74: 83-111.

12. Nústez, C., Santos, M., and Segura, M. 2009. Dry matter allocation and partitioning of four potato varieties (Solanum tuberosum L.) in Zipaquirá, Cundinamarca (Colombia). Revista Facultad Nacional de Agronomía. Medellín 62(1): 4823-4834.

13. Segura, M., Santos, M., and Nústez, C. 2006. Desarrollo fenológico de cuatro variedades de papa (Solanum tuberosum L.) en el Municipio de Zipaquirá (Cundinamarca). Fitotecnia Colombiana 6(2): 33-43.

14. Tekalign, T., and Hammes, P. S. 2005a. Growth and productivity of potato as influenced by cultivar and reproductive growth I. Stomatal cnductance, rate of transpiration, net photosynthesis, and dry matter production and allocation. Scientia Horticulturae 105(1): 13-27.

15. Tekalign, T., and Hammes, P. S. 2005b. Growth and productivity of potato as influenced by cultivar and reproductive growth II. Growth analysis, tuber yield and quality. Scientia Horticulturae 105(1):29-44. 